SCIENTIFIC REPORT

\title{
Comparison of corneal thickness measurements using Orbscan II, non-contact specular microscopy, and ultrasonic pachymetry in eyes after laser in situ keratomileusis
}

\author{
K Kawana, T Tokunaga, K Miyata, F Okamoto, T Kiuchi, T Oshika
}

Br J Ophthalmol 2004;88:466-468. doi: 10.1136/bjo.2003.030361

\begin{abstract}
Aims: To compare central corneal thickness measurements of three pachymetry devices in eyes after laser in situ keratomileusis (LASIK).

Methods: Central corneal thickness was measured in 203 eyes after myopic LASIK. Orbscan II scanning slit topography (Bausch \& Lomb), SP-2000P non-contact specular microscopy (Topcon), and ultrasonic pachymetry (Tomey) were used in this sequence.

Results: Three devices gave significantly different corneal thickness readings $(p<0.0001$, repeated measure analysis of variance). The measurements of Orbscan II (445.6 (SD 60.0) $\mu \mathrm{m}$ ) were significantly smaller than those of noncontact specular microscopy (467.9 (SD 40.2) $\mu \mathrm{m} ; \mathrm{p}<0.0001$, Tukey multiple comparison) and ultrasonic pachymetry (478.8 (SD 41.9) $\mu \mathrm{m} ; \mathrm{p}<0.0001$ ). The value obtained with SP-2000P non-contact specular microscopy was significantly smaller than that taken with ultrasonic pachymetry $(p<0.001)$. There were significant linear correlations between scanning slit topography and non-contact specular microscopy (Pearson's correlation coefficient $r=0.912$, $p<0.0001$ ), non-contact specular microscopy and ultrasonic pachymetry $(r=0.968, p<0.0001)$, and ultrasonic pachymetry and scanning slit topography $(r=0.933, p<0.0001)$. Conclusion: In post-LASIK eyes, Orbscan II scanning slit topography significantly underestimated corneal thickness. Non-contact specular microscopy gave smaller thickness readings than ultrasonic pachymetry, but these two units showed an excellent linear correlation.
\end{abstract}

\footnotetext{
C
} entral corneal thickness measurement is important in the pre and postoperative management of corneal surgical procedures such as laser in situ keratomileusis (LASIK). Ultrasonic pachymetry is currently the most commonly used technique to evaluate corneal thickness. Ultrasonic pachymetry, however, entails a direct contact of the probe onto the cornea, which may increase the risk of infection and corneal epithelium damage. Moreover, its accuracy is dependent on the perpendicularity of the probe's application to the cornea and reproducibility relies on precise probe placement on the corneal centre. Thus, examiner's experience can influence the reliability of measurements.

Recently, other sophisticated non-contact pachymetry instruments have been developed. The Orbscan II scanning slit topography (Bausch \& Lomb, Rochester, NY, USA) has multiple functions in the assessment of the cornea, including its thickness profile, anterior and posterior topography, elevation, and anterior chamber depth. The usefulness of this system has been reported previously. ${ }^{1-3}$ The SP-2000P non-contact specular microscopy (Topcon Corporation,
Tokyo, Japan) provides pachymetric measurements and specular microscopy simultaneously. ${ }^{45}$

Several studies have compared corneal thickness measurements between ultrasonic pachymetry and SP-2000 ${ }^{45}$ and Orbscan and ultrasonic pachymetry. ${ }^{26-14}$ To the best of our knowledge, there have been only two reports that compared these three methods. ${ }^{15} 16$ These studies, however, assessed three tests in normal subjects, and there has been no published comparison of these devices in eyes after refractive surgery. It has been reported that scanning slit topography underestimates corneal thickness in eyes after excimer laser keratorefractive surgery. ${ }^{74}$ Corneal pachymetry is important to evaluate the outcome of laser refractive surgical procedures, especially in candidates for enhancement surgery.

In this study, we compared central corneal thickness measurements in post-LASIK eyes obtained using three devices; Orbscan II scanning slit topography, SP-2000P non-contact specular microscopy, and ultrasonic pachymetry.

\section{SUBJECTS AND METHODS}

Central corneal thickness was determined in 203 post-LASIK eyes of 104 subjects with a mean age of 32.6 (SD 9.3) years old. Informed consent was obtained from all participants.

All measurements were taken at the same time of day, between 11:00 am and 2:00 pm, at least three hours after awakening. All eyes were examined with scanning slit topography (Orbscan II), non-contact specular microscopy (SP-2000P), and ultrasonic pachymetry (SP-2000, Tomey, Nagoya, Japan).

For Orbscan measurements, the patient's chin was placed on the chin rest and the forehead was pressed against the forehead strap. The patient was asked to look at the blinking fixation target, and the images were captured. The acoustic equivalent correction factor (0.92) was used to achieve equivalence with the ultrasonic evaluation as recommended by the manufacturer. After the Orbscan measurements, noncontact specular microscopic pachymetry was performed. The subject was positioned with his or her chin in a cup and forehead against a headband. Corneal thickness and endothelial cell density were measured. In this study, only central corneal thickness readings were used. Then, the cornea was anaesthetised with topical $0.4 \%$ oxybuprocaine hydrochloride and five consecutive measurements were made using ultrasonic pachymetry. The probe was sterilised and applied as perpendicular as possible on the central cornea.

\section{RESULTS}

The mean central corneal thickness of post-LASIK eyes is shown in table 1 . There was a significant variation in the measurement results among the three devices $(p<0.0001$, repeated measure analysis of variance). The measurements of Orbscan II were significantly smaller than those of noncontact specular microscopy and ultrasonic pachymetry 
Table 1 Central corneal thickness measurements in eyes after laser in situ keratomileusis

\begin{tabular}{lll}
\hline Devices & Mean $(\mathrm{SD})(\mu \mathrm{m})$ & Range $(\mu \mathrm{m})$ \\
\hline $\begin{array}{l}\text { Scanning slit topography } \\
\text { Non-contact specular }\end{array}$ & $445.6(60.0)$ & $270-575$ \\
microscopy & $467.9(40.2)$ & $387-566$ \\
Ultrasonic pachymetry & $478.8(41.9)$ & $395-575$ \\
\hline
\end{tabular}

$\mathrm{SD}$, standard deviation.

$(\mathrm{p}<0.0001$, Tukey multiple comparison). The value obtained with SP-2000P non-contact specular microscopy was significantly smaller than that taken with ultrasonic pachymetry $(\mathrm{p}<0.001)$.

The correlation between each test was evaluated. There were significant linear correlations between scanning slit topography and the non-contact specular microscopy (Pearson's correlation coefficient $r=0.912, \mathrm{p}<0.0001$; fig 1 ), non-contact specular microscopy and ultrasonic pachymetry $(r=0.968, \mathrm{p}<0.0001$; fig 2$)$, and ultrasonic pachymetry and scanning slit topography $(r=0.933, \mathrm{p}<0.0001$; fig 3$)$. The corneal thickness measurements by non-contact specular microscopy and ultrasonic pachymetry were highly compatible (fig 2), with the best fit line $y=6.0+1.01 \mathrm{x}, \mathrm{r}^{2}=0.938$.

\section{DISCUSSION}

As shown in the results, there was a significant variation in the corneal thickness readings among the three devices, and the measurements with Orbscan II scanning slit topography were significantly smaller than with the other two methods. In normal human eyes, Suzuki et al reported that corneal thickness readings were comparable between Orbscan II and ultrasonic pachymetry, and non-contact specular microscopy gave significantly smaller values. ${ }^{16}$ Módis et al reported similar findings in normal subjects. ${ }^{15}$ On the other hand, there have been reports that Orbscan II scanning slit topography significantly underestimated corneal thickness in eyes after excimer laser keratorefractive surgery, compared with ultrasonic pachymetry. ${ }^{74}$ These previous and current findings indicate that Orbscan II, when compared with ultrasonic pachymetry, gives comparable corneal thickness readings in normal eyes, but disproportionally lower readings in post-LASIK eyes.

It seems that corneal thickness measurements of Orbscan II scanning slit topography were calibrated in a normal

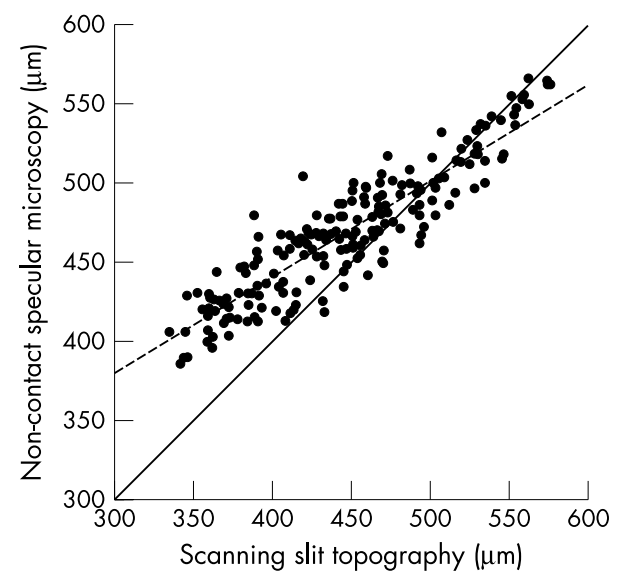

Figure 1 There was a significant correlation between corneal thickness measurements by scanning slit topography and non-contact specular microscopy (Pearson's correlation coefficient $r=0.912, p<0.001$ ). The best fit line $\left(y=195.5+0.61 x, r^{2}=0.832\right)$ is designated by the dotted line and the line of equivalence $(y=x)$, by the solid line.

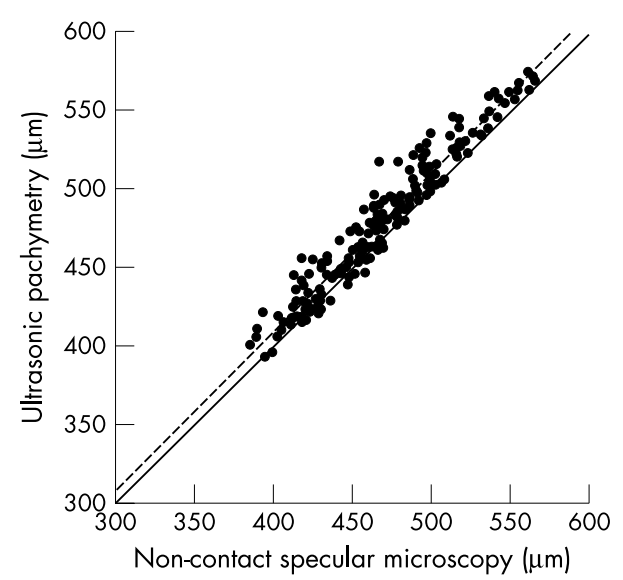

Figure 2 There was a significant correlation between corneal thickness measurements by non-contact specular microscopy and ultrasonic pachymetry (Pearson's correlation coefficient $r=0.968, p<0.001$ ). The best fit line $\left(y=6.0+1.01 x, r^{2}=0.938\right)$ is designated by the dotted line and the line of equivalence $(y=x)$, by the solid line.

population, where the acoustic equivalent correction factor (0.92) was obtained. In figure 3, there were good agreements between the measurements with Orbscan II and ultrasonic pachymetry in corneas with normal, preoperative range of thickness. On the other hand, it appears that the unit is not calibrated properly for thin corneas. For thinner corneas, there was a proportional and linear deviation from the acoustic values (fig 3) which can be compensated by using a certain slope factor for correction.

There may be several reasons to explain the discrepancy between Orbscan and ultrasonic pachymetric values after keratorefractive surgery. Because optical pachymetry as used by the Orbscan system is dependent on measurements of reflected light beams through the corneal tissue, when the corneal medium is not clear or has optical interfaces, the pathways of light rays may be interrupted. ${ }^{7}$ Factors which may influence optical pachymetry system such as Orbscan include all causes resulting in alterations in the optical quality of the cornea after excimer laser surgery: stromal haze, stromal interface, changes in refractive index, and postoperative modifications of the anterior corneal contour. ${ }^{14}$ Prisant et al reported that stromal haze seemed to play the most important role, because underestimation of Orbscan

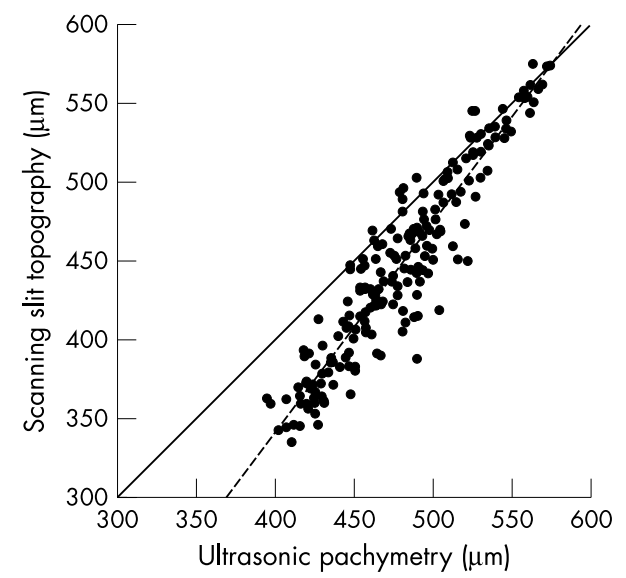

Figure 3 There was a significant correlation between corneal thickness measurements by ultrasonic pachymetry and scanning slit topography (Pearson's correlation coefficient $r=0.933, \mathrm{p}<0.001$ ). The best fit line $\left(y=-192.8+1.33 x, r^{2}=0.870\right)$ is designated by the dotted line and the line of equivalence $(y=x)$, by the solid line. 
pachymetry was much higher in the photorefractive keratectomy group than in the LASIK group..$^{14}$ Fakhry et al also demonstrated that the Orbscan II is an accurate pachymetry tool in normal eyes with results comparable with those of ultrasonic pachymetry, but in cases with haze after photorefractive keratectomy, the Orbscan readings were lower than the ultrasonic readings. ${ }^{9}$ Boscia et al reported that the Orbscan system offered less accurate corneal thickness measurements in eyes with clinically significant haze, though the repeatability of the measures was good. ${ }^{10}$ They suggested that the formation of new collagen (type III collagen) and vacuoles filled with proteoglycan debris (keratin sulfate), observed in eyes with haze, results in a high level of light scatter with an ensuing increase in the refraction index of the cornea, which compromised the optical pachymetry in these eyes. Although no case presented clinically significant haze in our subjects, the possibility exists that subtle and inhomogeneous changes in the corneal refractive index influenced Orbscan pachymetry measurements in eyes after LASIK.

Another consideration includes the shape factor. The natural and normal cornea is generally prolate, with steeper curvature centrally and relatively flatter peripherally. After LASIK, the curvature of the cornea is altered and tends to become more oblate. The reconstruction algorithms that construct the corneal elevation data in Orbscan, using lower order polynomials, effectively smooth small irregularities in the corneal surface. It is speculated that these algorithms may not properly apply to the corneas with non-physiological conditions such as post-LASIK eyes.

In our study, the mean corneal thickness measured by SP2000P non-contact specular microscopy was significantly smaller than the mean obtained by ultrasonic pachymetry, which agrees with the results in normal subjects as reported previously. $^{714}$ Other studies also report that non-contact specular microscopy provides corneal thickness measurements that are less than those of ultrasonic pachymetry. ${ }^{4}$ These differences are attributable to the different operating principles of the methods. Non-contact specular microscopy measurements depend on the reflection of light, and the ultrasonic pachymetry measurements depend on the reflection of ultrasonic from the anterior and posterior corneal surfaces. In ultrasonic pachymetry, the exact posterior reflection point is not known; it may be located between Descemet's membrane and the anterior chamber. It was proved that the ultrasonic probe can displace the 7 to $40 \mu \mathrm{m}$ thick tear film and thin the epithelium. ${ }^{17}$

Precise measurements of corneal thickness are important to evaluate the outcome of laser refractive surgical procedures, especially when considering enhancement surgery. Underestimation of corneal pachymetry may lead to exclusion of some of these patients and, in general, to an overly conservative treatment plan. ${ }^{14}$ Conversely, overestimation may increase the risk of corneal ectasia in ineligible patients. Our study indicated that three different pachymetry systems are not completely compatible in measuring central corneal thickness in eyes after excimer laser keratorefractive surgery.

\section{Authors' affiliations}

K Kawana, F Okamoto, T Kiuchi, T Oshika, Department of Ophthalmology, Institute of Clinical Medicine, University of Tsukuba, Ibaraki, Japan

T Tokunaga, K Miyata, Miyata Eye Hospital, Miyazaki, Japan

The authors have no commercial or proprietary interest in any of the companies, products, or methods described in this article.

Correspondence to: Dr T Oshika, Department of Ophthalmology, Institute of Clinical Medicine, University of Tsukuba, 1-1-1 Tennoudai, Tsukuba, Ibaraki, 305-8575 Japan; toshika@md.tsukuba.ac.jp

Accepted for publication 1 September 2003

\section{REFERENCES}

1 Lattimore MR, Kaupp S, Schallhorn S, et al. Orbscan pachymetry: implications of a repeated measures and diurnal variation analysis. Ophthalmology 1999; 106:977-81.

2 Yaylali V, Kaufman SC, Thompson HW. Corneal thickness measurements with the Orbscan Topography System and ultrasonic pachymetry. J Cataract Refract Surg 1997;23:1345-50.

3 Liu Z, Huang AJ, Pflugfelder SC. Evaluation of corneal thickness and topography in normal eyes using the Orbscan corneal topography system. Br J Ophthalmol 1999;83:774-8.

4 Bovelle R, Kaufman SC, Thompson HW, et al. Corneal thickness measurements with the Topcon SP-2000P specular microscope and an ultrasound pachymeter. Arch Ophthalmol 1999;117:868-70.

5 Módis L, Langenbucher A, Seitz B. Corneal thickness measurements with contact and noncontact specular microscopic and ultrasonic pachymetry. Am J Ophthalmol 2001;132:517-21.

6 Marsich MW, Bullimore MA. The repeatability of corneal thickness measures. Cornea 2000; 19:792-5.

7 Iskander NG, Anderson Penno E, Peters NT, et al. Accuracy of Orbscan pachymetry measurements and DHG ultrasound pachymetry in primary laser in situ keratomileusis and LASIK enhancement procedures. J Cataract Refract Surg 2001;27:681-5.

8 Chakrabarti HS, Craig JP, Brahma A, et al. Comparison of corneal thickness measurements using ultrasound and Orbscan slit-scanning topography in normal and post-LASIK eyes. J Cataract Refract Surg 2001;27:1823-8.

9 Fakhry MA, Artola A, Belda Jl, et al. Comparison of corneal pachymetry using ultrasound and Orbscan II. J Cataract Refract Surg 2002;28:248-52.

10 Boscia F, La Tegola MG, Alessio G, et al. Accuracy of Orbscan optical pachymetry in corneas with haze. J Cataract Refract Surg 2002;28:253-8.

11 Wong AC, Wong CC, Yuen NS, et al. Correlational study of central corneal thickness measurements on Hong Kong Chinese using optical coherence tomography, Orbscan and ultrasound pachymetry. Eye 2002;16:715-21.

12 Giraldez Fernandez MJ, Diaz Rey A, Cervino A, et al. A comparison of two pachymetric systems: slit-scanning and ultrasonic. CLAO J 2002;28:221-3.

13 Gonzalez-Meijome JM, Cervino A, Yebra-Pimentel E, et al. Central and peripheral corneal thickness measurement with Orbscan II and topographical ultrasound pachymetry. J Cataract Refract Surg 2003;29:125-32.

14 Prisant O, Calderon N, Chastang P, et al. Reliability of pachymetric measurements using Orbscan after excimer refractive surgery. Ophthalmology 2003;110:511-15.

15 Módis L, Langenbucher A, Seitz B. Scanning-slit and specular microscopic pachymetry in comparison with ultrasonic determination of corneal thickness. Cornea 2001;20:711-14.

16 Suzuki S, Oshika T, Oki K, et al. Corneal thickness measurements using scanning-slit corneal topography and noncontact specular microscopy versus ultrasonic pachymetry. J Cataract Refract Surg 2003;29:1313-18.

17 Nissen J, Hjortdal JO, Ehlers N, et al. A clinical comparison of optical and ultrasonic pachometry. Acta Ophthalmol (Copenh) 1991;69:659-63. 